\title{
Safety of two-hour intermittent intravenous infusions of tacrolimus in the allogeneic hematopoietic stem cell transplantation unit
}

Alexander J. S. Bacopoulos, BSc, PharmD, ACPR ${ }^{1,2}$; Lina Ho, BScPhm, ACPR ${ }^{1,2,3}$; Anjie Yang, BScPhm, ACPR ${ }^{1,2}$; Pamela Ng, BScPhm²; Celina Dara, BScPhm, PharmD, ACPR ${ }^{2}$; David Loach, $\mathrm{MD}^{3,4}$; Uday Deotare, MD, $\mathrm{DM}^{3,4}$; Fotios V. Michelis, $\mathrm{MD}, \mathrm{PhD}^{3,4}$; Auro Viswabandya, $\mathrm{MD}$, $\mathrm{DM}^{3,4}$; Dennis D. Kim, MD, PhD ${ }^{3,4}$; Jeffrey H. Lipton, $\mathrm{MD}, \mathrm{PhD}^{3,4}$; Hans $\mathrm{A}$. Messner, $\mathrm{MD}, \mathrm{PhD}^{3,4}$; Santhosh Thyagu, MD, $\mathrm{DM}^{3,4}$

\author{
${ }^{1}$ Leslie Dan Faculty of Pharmacy, University of Toronto \\ ${ }^{2}$ Department of Pharmacy, University Health Network \\ ${ }^{3}$ Allogeneic Blood and Marrow Transplant Program, University Health Network \\ ${ }^{4}$ Faculty of Medicine, University of Toronto
}

Key words: tacrolimus, allogeneic hematopoietic stem cell transplantation; HSCT, infusion time, nephrotoxicity, neurotoxicity, infusion-related reactions; IRR 


\begin{abstract}
Background: Administering intravenous (IV) tacrolimus by 24-hour continuous IV infusion (CIV), as recommended by the product monograph, poses significant logistical challenges in the allogeneic hematopoietic stem cell transplantation (HSCT) unit because it requires a dedicated central venous catheter lumen. Consequently, at our institution, tacrolimus has been administered via two-hour intermittent IV infusions (IIV) every twelve hours in the HSCT unit. Administration by IIV is not the standard of practice and shorter infusion times are cautioned due to higher rates of nephrotoxicity, neurotoxicity and infusion-related reactions (IRRs), although there is a paucity of data to support this claim. The primary objective of this retrospective study was to evaluate the safety of a two-hour IIV of tacrolimus in an adult HSCT population. Efficacy was evaluated as a secondary endpoint.
\end{abstract}

Methods and Patients: We performed a retrospective chart review of all patients who received IV tacrolimus at our institution from January 2002 - January 2016. We reviewed 104 patients who received 118 tacrolimus treatment courses by IIV (TTC) [median number of doses per TTC=22, range 1 158 , interquartile range $(I Q R)=28$ ]. Primary outcomes collected include rates of nephrotoxicity, neurotoxicity and IRRs that occurred during TTC. The incidence of acute graft-versus-host disease (aGVHD) and disease relapse within 180 days of transplant were collected to evaluate efficacy.

Results and Discussion: There were sixteen incidences (13.6\%) of nephrotoxicity, defined as a doubling of serum creatinine from baseline. Nephrotoxicity resolved in all but six (5.1\%) cases. Precipitating factor for nephrotoxicity unrelated to tacrolimus were identified by the physician in all six cases. There were 40 incidences (34.5\%) of neurotoxicity [seizure, posterior reversible encephalopathy syndrome (PRES), tremor, paresthesia, visual disturbance], of which, eight (6.8\%) were considered serious (seizure and/or PRES). All neurotoxicity reverted to baseline or resolved completely. One grade 2 infusion reaction occurred and resolved without discontinuation of tacrolimus. In the subset of patients who received tacrolimus for the prevention of aGVHD $(n=41)$, seven patients $(17.1 \%)$ developed grade II - IV aGVHD. Nine patients (8.7\%) experienced relapse of their disease.

Conclusions: We propose that a two-hour IIV of tacrolimus is a safe method of administration in the adult HSCT setting. Additional safety and efficacy data from other institutions will provide external validity to this conclusion. 


\section{Background}

Calcineurin inhibitors, cyclosporine (CsA) and tacrolimus, are the drugs of choice used in allogeneic hematopoietic stem cell transplantation (HSCT) to prevent or treat graft-versus-host disease $(G V H D)^{1,2}$. At our institution, CsA is used first-line. Tacrolimus is reserved as a secondline option in cases where intolerable adverse events (AE) to CsA occur.

Achieving the desired degree of immunosuppression following HSCT is challenging. While aggressive immunosuppression is desirable to decrease the incidence of GVHD, it also suppresses the graft-versus-tumour effect, or anti-cancer activity, of the transplant. Suppression of the graft-versus-tumour effect can lead to relapse of disease ${ }^{2}$.

The Prograf ${ }^{\circledast}$ (tacrolimus) monograph recommends intravenous (IV) administration via 24-hour continuous infusion (CIV) when the medication cannot be administered orally ${ }^{3}$.

HSCT patients routinely require numerous essential IV medications and blood products. At our institution, all HSCT patients receive a double-lumen central venous catheter for IV access. Dedicating one of these lumens to a CIV of tacrolimus for days to weeks becomes logistically challenging when trying to accommodate multiple medications and blood products. Similar challenges at other institutions have prompted research on the safety and efficacy of tacrolimus via shortened infusion durations ${ }^{4,5}$.

The nursing IV guidelines at our institution refer to the Prograf ${ }^{\circledR}$ monograph and recommend administration of tacrolimus by 24-hour CIV and, as an alternative, administration via four-hour intermittent IV infusions (IIV) every twelve hours. The guidelines caution the use of the four-hour infusion, associating shorter infusions with higher rates of nephrotoxicity, 
neurotoxicity and infusion-related reactions (IRR). However, there is a paucity of published data to support the precaution.

Although there are no reports in the literature describing experience in an adult population, a two-hour IIV infusion of tacrolimus has been proposed as a safe and efficacious practice for the prevention of GVHD in the pediatric setting ${ }^{4}$. For the last fifteen years or more, in our institution, tacrolimus has been administered as a two-hour IIV every twelve hours in the allogeneic HSCT unit.

The primary objective of this study was to evaluate the safety of a two-hour IIV of tacrolimus in the HSCT population and to report the outcomes from experience within our institution. The secondary objective was to evaluate its efficacy in preventing acute GVHD (aGVHD).

\section{Methods}

\section{Patient Population}

We performed a retrospective review of the paper charts and electronic patient records (EPR) of all patients who received IV tacrolimus between January 1, 2002 and January 1, 2016. All patients received IV tacrolimus via two-hour IIV as per institutional standard of practice. A total of 104 patients were identified and analyzed. No patients were excluded. A minority of patients received IV tacrolimus on two separate hospital admissions for a total of 118 IV tacrolimus treatment courses (TTC). 


\section{Safety Outcomes}

Safety outcomes were reported as a percent incidence of 118 TTCs. The primary safety outcomes were nephrotoxicity, neurotoxicity and IRRs. Nephrotoxicity was defined as a doubling of serum creatinine (SCr) during IV TTC from baseline ${ }^{4,6-8}$. Neurotoxicity was defined as the new onset occurrence of seizure $\mathrm{e}^{3,4,6-8}$, posterior reversible encephalopathy syndrome (PRES) as confirmed by magnetic resonance imaging (MRI) of the brain ${ }^{3,4}$, tremor $^{3}$, paresthesia ${ }^{3}$ or visual disturbance ${ }^{3}$ during IV tacrolimus administration. Seizures and PRES were considered to be severe neurotoxicity. IRRs were graded according to the Common Terminology Criteria for Adverse Events Version $4.0^{9}$.

Other safety outcomes evaluated include commonly reported AEs of tacrolimus: hypertension, hyperglycemia, hypomagnesemia and hyperbilirubinemia ${ }^{3}$. Hypertension was defined as the requirement for a new antihypertensive medication prescription or an increase in the dosage of an existing antihypertensive medication ${ }^{4,6-8}$. Similarly, hyperglycemia was defined as the requirement for a new insulin prescription or an increase in insulin requirements ${ }^{6-8}$. We defined hypomagnesemia as serum magnesium $(\mathrm{Mg}$ ) less than 0.7 $\mathrm{mmol} / \mathrm{L}$ (our institutional threshold below which $\mathrm{Mg}$ supplementation is recommended) or the requirement for $\mathrm{Mg}$ supplementation to keep serum $\mathrm{Mg}$ above this value ${ }^{4}$. Hyperbilirubinemia was defined as a peak serum bilirubin level greater than $22 \mu \mathrm{mol} / \mathrm{L}$ (our institutional upper limit of normal) and in cases where the baseline serum bilirubin was greater than $22 \mu \mathrm{mol} / \mathrm{L}$, any worsening of this value. 
The incidence of two HSCT-related AEs, hepatic sinusoidal obstruction syndrome (SOS) and reactivation of cytomegalovirus (CMV), were also reported as exploratory outcomes. SOS was defined in accordance with the Baltimore criteria and reported if IV tacrolimus was initiated within 28 days of $\mathrm{HSCT}^{10}$. Our institution initiates pre-emptive antiviral therapy for CMV infection when the CMV blood polymerase chain reaction (PCR) value exceeds 200 units/mL for very high-risk patients, $500 \mathrm{IU} / \mathrm{mL}$ for high-risk patients and 1000 units/mL for lowrisk patients. However, this test has only been the institutional standard of practice for the past decade. Therefore, $\mathrm{CMV}$ reactivation was defined as the requirement for pre-emptive antiviral therapy following IV tacrolimus therapy within 180 days of HSCT, regardless of the diagnostic test or yield.

\section{Efficacy Outcomes}

The primary measure of efficacy was incidence of grade II - IV aGVHD within 180 days of HSCT as defined by the Centre for International Blood and Marrow Transplant Research

(CIBMTR) grading system. Acute GVHD incidence was reported for the patients who received IV tacrolimus for the prevention of aGVHD $(n=43)$

Relapse of disease within 180 days of HSCT was also reported. Relapse was identified by physician diagnosis in the patient notes and by reviewing bone marrow biopsy reports.

\section{Statistical Analysis}

Data were reported as event incidence per total number of possible event occurrences. The data reported are observational in nature and no comparative analysis was performed. 


\section{Results}

\section{Patient Characteristics}

Patient characteristics are summarized in Table 1. The median age on day of HSCT was 48 years [range $17-69$; interquartile range (IQR) 19.25]. One patient did not receive HSCT and therefore, age on day of first IV tacrolimus dose was recorded instead. Fifty-nine patients (56.7\%) were women and $45(43.3 \%)$ were men. The most common diagnosis was acute myeloid leukemia (AML; $n=33 ; 31.7 \%$ ), followed by acute lymphoblastic leukemia $(A L L ; n=11$; $10.6 \%$ ), myelofibrosis ( $n=11 ; 10.6 \%)$, non-malignant anemias ( $n=14 ; 13.5 \%)$, chronic myeloid leukemia (CML; $n=10 ; 9.6 \%)$, lymphoma $(n=8 ; 7.7 \%)$, myelodysplastic syndrome (MDS; $n=7$; $6.7 \%)$, chronic lymphoblastic leukemia (CLL; $n=5 ; 4.8 \%$ ) and other (mixed acute leukemia, multiple myeloma, myelomonocytic leukemia, agnogenic myeloid metaplasia, Waldenstrom's macgroglobulinemia) ( $n=5 ; 4.8 \%)$.

\section{Allogeneic Hematopoietic Stem Cell Transplantation (HSCT) Characteristics}

HSCT and donor characteristics are summarized in Table 1. Of the 103 patients who received HSCT, 43 were from matched-related donors (41.3\%), two were from mismatchedrelated donors (1.9\%), 43 were from matched-unrelated donors (41.3\%) and fifteen were from mismatched-unrelated donors (14.4\%).

Approximately half of all patients received myeloablative (MA) conditioning $(n=51$; 49.5\%) and the remaining half received reduced intensity conditioning ( $R I C)(n=52 ; 50.5 \%)$. The most common conditioning regimen was fludarabine (Flu), busulfan (Bu) and total body irradiation (TBI) at a dose of $200 \mathrm{cGy}(n=32,31.1 \%)$, followed by Flu + Bu + TBI $400 \mathrm{cGy}(\mathrm{n}=22$; 
21.4\%), Cyclophosphamide (Cy) + TBI $(n=20,19.4 \%), B u+C y(n=9,8.7 \%)$, Flu + melphalan $(n=$ $5 ; 4.9 \%)$, Flu + Cy $(n=5 ; 4.9 \%), C y+$ cytarabine + TBI $(n=4 ; 3.9 \%), C y(n=3 ; 2.9 \%)$, or other (etoposide $+\mathrm{Bu}$, etoposide + TBI) $(n=2 ; 1.9 \%)$. Data were missing for one HSCT $(1.0 \%)$.

The most common GVHD prophylaxis regimen used was a combination of CsA and mycophenolate mofetil (MF) ( $n=33 ; 32.0 \%)$, followed by CsA + methotrexate (MTX) ( $n=27$; $26.2 \%)$, alemtuzumab + CsA $(n=25 ; 25.5 \%)$, tacrolimus + MTX $(n=5 ; 4.9 \%)$, alemtuzumab + tacrolimus ( $n=4 ; 3.9 \%$ ), antithymocyte globulin (ATG) + post-transplant cyclophosphamide $($ PTCy) + CsA ( $=3 ; 2.9 \%)$ and other (ATG + PTCy + MTX, alemtuzumab + tacrolimus + MF, alemtuzumab $+C s A+M F, M F+$ tacrolimus $)(n=4 ; 3.9 \%)$. Data were missing for one HSCT (1.0\%).

Tacrolimus Treatment Course (TTC) Characteristics

TTC characteristics are summarized in Table 2. There were a total of 118 TTCs. The most common indication was treatment of GVHD ( $n=73 ; 61.9 \%)$, followed by prevention of GHVD ( $n=43 ; 36.4 \%$ ) and other (hemophagocytic lymphohistiocytosis, aplastic anemia) ( $n=2$; 1.7\%). The median number of doses per TTC was 22 (range $1-158 ;$ IQR 28). Most TTCs were administered following an AE to first-line CsA $(n=94 ; 79.7 \%)$. Ten TTCs were part of the initial GVHD prophylactic regimen as per protocol (8.5\%). Five TTCs were administered instead of CsA because of patient-specific factors (4.2\%). One TTC was selected as treatment for aplastic anemia (0.8\%). Data were missing for eight TTCs (6.8\%). Of the 94 TTCs administered following an $A E$ to CSA, the most common AE was neurotoxicity $(n=45 ; 48.4 \%)$, followed by red blood cell fragmentation $(n=15 ; 16.1 \%)$, gum hyperplasia $(n=14 ; 15.1 \%)$, hepatotoxicity $(n=3$; 
$3.2 \%)$, skin toxicity $(n=3 ; 3.2 \%)$, nephrotoxicity $(n=2 ; 2.2 \%)$, more than one $A E(n=2 ; 2.2 \%)$ or other AEs (anorexia, bradycardia, cholestasis, elevated lactose dehydrogenase, pruritis) ( $n=5$; $5.4 \%)$

Safety Outcomes

The incidences of safety outcomes are summarized in Figure 1.

Nephrotoxicity

The median serum creatinine ( $\mathrm{SCr}$ ) at baseline was $75 \mathrm{mmol} / \mathrm{L}$ (range $25-472$; IQR 46.75). The median peak SCr during TTC was $107 \mathrm{mmol} / \mathrm{L}$ (range $44-472$; IQR 69.5). In fortyone TTCs (34.7\%), a peak SCr that exceeded twice their baseline value was documented. Except for six cases, all patients' SCr returned to below twice the baseline value. In each of these six cases, precipitating factors for the renal insult were identified by the physician. These factors included: enterocolitis, renal injury secondary to conditioning chemotherapy, renal injury secondary to foscarnet, and septic shock secondary to pneumonia. One TTC resulted in the requirement for dialysis $(0.8 \%)$.

\section{Neurotoxicity}

Severe

The incidence of any neurotoxicity during TTC was $34.8 \%(n=41)$. Four new-onset seizures occurred (3.9\%). In the first two cases, the seizures were attributed to ongoing cerebral demyelination/PRES resulting from previous CSA therapy by the physician. In the third case, the seizure was attributed to a combination of hyperglycemia as well as a subdural 
hematoma by the physician. The seizure in the fourth case occurred following the twentieth IV tacrolimus dose. Tacrolimus was discontinued and no subsequent seizure was documented during that hospital admission. PRES was documented during seven TTCs (5.9\%).

Non-severe

The incidence of visual disturbances was $17.9 \%(n=21)$. One occurrence of blurry vision was followed by a new diagnosis of cataracts. Another episode of blurry vision was documented following a fall and a subsequent large hematoma in the periorbital area. A third occurrence of blurry vision was attributed to the use of multiple ophthalmic ointments. The incidence of paresthesia was $5.1 \%(n=6)$ and the incidence of tremor was $12.8 \%(n=15)$. One case of tremor was attributed to an extrapyramidal symptom from concurrent olanzapine usage. All cases of tremors occurred in the setting of concurrent hypomagnesemia. Data for non-severe neurotoxicity (visual disturbance, tremor, paresthesia) were missing for one patient whose paper chart was unavailable.

All neurotoxicity, regardless of severity, either reverted to baseline or resolved completely.

Infusion Related Reactions (IRR)

One IRR occurred $(0.8 \%)$. It was a grade 2 bradycardic episode ${ }^{9}$ that occurred during the eighth dose of IV tacrolimus in which the patient's heart rate lowered to 38 beats per minute. The IV tacrolimus infusion was stopped and heart rate returned to baseline the following day. Tacrolimus therapy was changed to the oral route the following day for an unrelated reason. Data were missing for one TTC due to the unavailability of the paper chart. 


\section{Other Adverse Effects (AEs)}

Hypertension and hyperglycemia both occurred during 25 TTCs (21.2\%), respectively. Hypomagnesemia occurred during 102 TTCs (86.4\%). Median serum bilirubin at baseline was $18 \mu \mathrm{mol} / \mathrm{L}$ (range 3 - 781; IQR 24.75). Median peak bilirubin during TTC was $34 \mu \mathrm{mol} / \mathrm{L}$ (range, 6 -842; IQR 59). Hyperbilirubinemia occurred in 50\% $(n=59)$ of TTCs.

\section{$\underline{\text { HSCT-Related AES }}$}

The incidence of SOS was $51 \%(n=6)$ and the incidence of CMV reactivation was $20.5 \%(n=24)$.

\section{Efficacy Outcomes}

The incidences of efficacy outcomes are summarized in Figure 2.

\section{Acute Graft-Versus-Host Disease (aGVHD)}

Of the 43 patients who received IV tacrolimus for the prevention of GVHD, fifteen (25.9\%) developed grade II - IV aGVHD. Three patients (7.0\%) developed grade III aGVHD and five patients (11.6\%) developed grade IV aGVHD. For two patients who developed grade II - IV aGVHD, the exact grade could not be determined due to missing data. Thirteen of the 43 patients did not have a complete follow-up period of 180 days.

\section{$\underline{\text { Relapse }}$}

Nine patients (8.7\%) experienced a relapse of their disease. Thirteen patients (12.5\%) were excluded from relapse analysis because they began IV tacrolimus therapy beyond 180 days post-HSCT. Forty-nine patients (47.1\%) did not have a complete follow-up period of 180 days. 


\section{Discussion}

This is the first study to address the safety of a two-hour intermittent infusion of IV tacrolimus in an adult population. The research question has been evaluated in a pediatric population by Skeens et al in $2012^{4}$, who concluded that a two-hour infusion of tacrolimus could be administered without increased toxicity or decreased efficacy in preventing GVHD.

There exists a considerable amount of safety and efficacy data for the administration of tacrolimus via CIV following $\mathrm{HSCT}^{6-8}$. All of these studies involve the initiation of tacrolimus as the first line GVHD prophylactic agent and follow the patients for a set duration of time postHSCT. This is a key contrast to our study, in which the majority of patients received tacrolimus as the second line agent following an AE from CSA. Our timeframe for the primary safety endpoints was the duration of the TTC which, in most cases, was a much shorter duration than studied in the CIV trials and therefore does not allow for direct comparison of safety outcome incidences. In addition to experiencing an AE while on CsA therapy, all patients in our study who received IV tacrolimus had a contraindication to oral therapy. The patients in this study can, therefore, be considered to have much higher morbidity at baseline than the populations studied in the CIV trials.

The AEs reported in this study were deemed to be of low clinical significance by the expert group of staff HSCT physicians at our institution. A two-hour IIV of tacrolimus did not independently precipitate irreversible nephrotoxicity in any of the patients studied. All cases of neurotoxicity resolved or reverted to baseline and only one IRR occurred which did not impact the patient's clinical course or outcomes. 
The most common AEs that occurred were well-known and commonly reported AEs of tacrolimus: hypomagnesemia, hyperbilirubinemia, hypertension and hyperglycemia. Hypertension, hyperglycemia and hypomagnesemia were all managed with routine supportive medications. Hyperbilirubinemia and hepatotoxicity, in particular, could be confounded by various factors in the HSCT population. Coexisting GVHD complicates the task of detecting tacrolimus-induced hepatotoxicity. HSCT patients are also exposed to numerous hepatotoxic drugs in their conditioning regimens and supportive medications, such as CsA, methotrexate, steroids, antivirals, antibiotics and antifungals ${ }^{11}$. Of note, our institution does not prescribe routine prophylactic antivirals to prevent CMV reactivation in HSCT patients. The threshold value that we chose for defining hypomagnesemia in this study differs from the thresholds reported in the literature ${ }^{4,6-8}$. Our institutional threshold $\mathrm{Mg}$ level for supplementation is much higher than some of the definitions utilized in the literature. If a $\mathrm{Mg}$ level of $0.6 \mathrm{mmol} / \mathrm{L}$ is used, similar to the threshold value used in the CIV trials, the incidence of hypomagnesemia in our study falls to $54.2 \%$.

The incidence of grade II - IV aGVHD following HSCT varies greatly in the literature. There is a variety of factors that influence the development of GVHD including, but not limited to, GVHD prophylaxis regimen, major histocompatibility complex matching, donor stem cell source and age $\mathrm{e}^{12-14}$. A recent study evaluating over 5000 HSCTs from the CIBMTR reported the incidence of grade II - IV aGVHD in sibling donor transplants and unrelated matched donor transplants to be $39 \%$ and $59 \%$, respectively ${ }^{12}$. While our study is not designed to be comparative in nature, the incidence of grade II - IV aGVHD reported here appears to be consistent with the ranges that exist in the literature ${ }^{15}$. 
Almost half of the patients who received tacrolimus for the prevention of GVHD in our study were lost to follow-up prior to the predefined 180-day follow-up period, which does lower the strength of the efficacy data. Many patients lost to follow-up simply did not have any clinic notes in the EPR beyond 180 days post-HSCT and the reason for being lost to follow-up could not be determined. Due to the nature of their disease and transplant-related mortality ${ }^{12}$, many patients, unfortunately, passed away prior to day 180.

\section{Limitations of the Study}

The retrospective nature of this study lends itself to a number of limitations. Many of the common AEs of tacrolimus are shared by $\operatorname{CsA}^{3,16}$. The definitions of safety outcomes were developed in order to capture only new or worsening AEs in order to minimize confounding from pre-existing CsA toxicity. Much of the data were collected from daily nursing notes in the paper chart and electronic discharge summaries from the EPR. These reports are only as complete as the quality of the documentation and it is more than likely that minor AEs such as tremors or flushing were underestimated.

Limitations aside, the results collected from this study strongly support the claim that a two-hour IIV of tacrolimus is a safe practice and not associated with increased toxicity. We conclude that a two-hour intermittent IV infusion of tacrolimus is a safe method of administration. Efficacy in preventing aGVHD does not appear to be compromised with a twohour infusion either. Further safety and efficacy data from other centres would increase the external validity of our findings. 


\section{Acknowledgements}

The authors would like to extend their gratitude to Vanessa Alexander, Samantha Polito, Saba

Chowdhry, Wallace Lam and Tony Panzarella for their help and counsel in conducting this study.

\section{Financial Disclosure}

The authors have nothing to disclose.

\section{Conflicts of Interest}

There are no conflicts of interest to report. 


\section{References}

1. Ruutu T, Gratwohl a, de Witte T, et al. Prophylaxis and treatment of GVHD: EBMT-ELN working group recommendations for a standardized practice. Bone Marrow Transplant. 2014;49(2):168-173. doi:10.1038/bmt.2013.107.

2. Negrin RS. Graft-versus-host disease versus graft-versus-leukemia. Hematology Am Soc Hematol Educ Program. 2015;2015(1):225-230. doi:10.1182/asheducation-2015.1.225.

3. Astellas Pharma Canada Inc. Product Monograph: Prograf. Markham, ON; 2011.

4. Skeens M, Pai V, Garee A, et al. Twice daily i.v. bolus tacrolimus infusion for GVHD prophylaxis in children undergoing stem cell transplantation. Bone Marrow Transplant. 2012;47(11):1415-1418. doi:10.1038/bmt.2012.59.

5. F.N. Hayek, H. Al-Ghawi, J. Allen, T. Baize, L. Mudd, A. Mehta, S. Soni, A. Cheerva, G.P. Herzig RHH. Twice-daily intravenous bolus tacrolimus infusion for acute graft-vs-host disease prophylaxis. Biol Blood Marrow Transplant. 2005;11(2 Supplement 1):45. http://www.ncbi.nlm.nih.gov/pubmed/26637726.

6. Fay JW, Wingard JR, Antin JH, et al. FK506 (Tacrolimus) monotherapy for prevention of graft-versus-host disease after histocompatible sibling allogenic bone marrow transplantation. Blood. 1996;87(8):3514-3519.

http://www.ncbi.nlm.nih.gov/pubmed/8605372.

7. Woo M, Przepiorka D, Ippoliti C, et al. Toxicities of tacrolimus and cyclosporin A after allogeneic blood stem cell transplantation. Bone Marrow Transplant. 1997;20(12):10951098. doi:10.1038/sj.bmt.1701027.

8. Yanik G, Levine JE, Ratanatharathorn V, Dunn R, Ferrara J, Hutchinson RJ. Tacrolimus (FK506) and methotrexate as prophylaxis for acute graft-versus-host disease in pediatric allogeneic stem cell transplantation. Bone Marrow Transplant. 2000;26(2):161-167. doi:10.1038/sj.bmt.1702472.

9. National Cancer Instutution. Common Terminology Criteria for Adverse Events (CTCAE) Common Terminology Criteria for Adverse Events v4.0 (CTCAE). 2009;2009. https://evs.nci.nih.gov/ftp1/CTCAE/CTCAE_4.03_2010-06-14_QuickReference_5x7.pdf.

10. Jones RJ, Lee KSR, Beschorner WE, et al. Venoocclusive Disease of the Liver following Bone Marrow Transplantation. Transplantation. 1987;44(6):778-783.

11. Ozdoğan O, Ratip S, Ahdab Y Al, et al. Causes and risk factors for liver injury following bone marrow transplantation. J Clin Gastroenterol. 2003;36(5):421-426.

12. Jagasia M, Arora M, Flowers MED, et al. Risk factors for acute GVHD and survival after hematopoietic cell transplantation. Blood. 2013;119(1):296-308. doi:10.1182/blood2011-06-364265.An.

13. Nassereddine S, Rafei H, Elbahesh E, Tabbara I. Acute Graft Versus Host Disease: A Comprehensive Review. Anticancer Res. 2017;37(4):1547-1555. 
doi:10.21873/anticanres.11483.

14. Choi SW, Levine JE FJ. Pathogenesis and Management of Graft versus Host Disease Sung. Immunol Allergy Clin North Am. 2010;30(1):75-101. doi:10.1016/j.iac.2009.10.001.

15. Sullivan KM. Graft-vs-host disease. In: Blume KG, Forman SJ, Appel-baum FR, eds. Thomas' Haematopoietic Cell Transplantation. Oxord, UK: Blackwell Publishing Ltd.; 2004:635-664.

16. Novartis Pharmaceuticals Canada Inc. Product Monograph: Neoral. Dorval, QC; 2015. 
Table 1. Patient, Donor and Transplant Characteristics

\begin{tabular}{|c|c|}
\hline Number of patients & 104 \\
\hline Median age on day of transplant, years (range; IQR) & $48(17-69 ; 19.25)$ \\
\hline \multicolumn{2}{|l|}{ Gender, no. (\%) } \\
\hline Female & $59(56.7)$ \\
\hline Male & $45(43.3)$ \\
\hline \multicolumn{2}{|l|}{ Diagnosis, no. (\%) } \\
\hline Acute myeloid leukemia & $33(31.7)$ \\
\hline Acute lymphoblastic leukemia & $11(10.7)$ \\
\hline Non-malignant anemias & $14(13.5)$ \\
\hline Myelofibrosis & $11(10.7)$ \\
\hline Chronic myeloid leukemia & $10(9.6)$ \\
\hline Lymphoma & $8(7.7)$ \\
\hline Myelodysplastic syndrome & $7(6.7)$ \\
\hline Chronic lymphoblastic leukemia & $5(4.8)$ \\
\hline Other* & $5(4.8)$ \\
\hline \multicolumn{2}{|l|}{ Donor type, no. (\%) } \\
\hline Matched-related & $43(41.3)$ \\
\hline Mismatched-related & $2(1.9)$ \\
\hline Matched-unrelated & $43(41.3)$ \\
\hline Mismatched-unrelated & $15(14.4)$ \\
\hline \multicolumn{2}{|l|}{ Conditioning regimen, no. (\%) } \\
\hline $\mathrm{Flu}+\mathrm{Bu}+\mathrm{TBI}(200 \mathrm{cGy})$ & $32(31.1)$ \\
\hline $\mathrm{Flu}+\mathrm{Bu}+\mathrm{TBI}(400 \mathrm{cGy})$ & $22(21.4)$ \\
\hline $\mathrm{Cy}+\mathrm{TBI}$ & $20(19.4)$ \\
\hline $\mathrm{Bu}+\mathrm{Cy}$ & $9(8.7)$ \\
\hline Flu + melphalan & $5(4.9)$ \\
\hline $\mathrm{Flu}+\mathrm{Cy}$ & $5(4.9)$ \\
\hline Cy + Ara-C + TBI (500 cGy) & $4(3.9)$ \\
\hline Cy & $3(2.9)$ \\
\hline$B u+V P-16$ & $1(1.0)$ \\
\hline VP-16 + TBI (1200 cGy) & $1(1.0)$ \\
\hline Missing data & $1(1.0)$ \\
\hline \multicolumn{2}{|l|}{ GVHD prophylaxis, no. (\%) } \\
\hline $\mathrm{CsA}+\mathrm{MF}$ & $33(32.0)$ \\
\hline CsA + MTX & $27(26.2)$ \\
\hline Alemtuzumab + CsA & $26(25.2)$ \\
\hline Tacrolimus + MTX & $5(4.9)$ \\
\hline Alemtuzumab + tacrolimus & $4(3.9)$ \\
\hline ATG + PTCy + CsA & $3(2.9)$ \\
\hline Other & $4(3.9)$ \\
\hline Missing data & $1(1.0)$ \\
\hline
\end{tabular}


Table 2. Tacrolimus Treatment Course (TTC) Characteristics

\begin{tabular}{lc}
\hline $\begin{array}{l}\text { No. of TTCs } \\
\text { Median tacrolimus doses per TTC, no. (range; IQR) }\end{array}$ & 118 \\
$\begin{array}{l}\text { Indication for tacrolimus, no. (\%) } \\
\text { Treatment of GVHD }\end{array}$ & $73(61.9)$ \\
Prevention of GVHD & $43(36.4)$ \\
Other* & $2(1.7)$ \\
Reason for using tacrolimus (instead of CsA), no. (\%) & $94(79.7)$ \\
Adverse event from CsA & $45(48.4)$ \\
Neurotoxicity & $15(16.1)$ \\
RBC fragmentation & $14(15.1)$ \\
Gum hyperplasia & $5(5.4)$ \\
Hypertension & $3(3.2)$ \\
Hepatotoxicity & $3(3.2)$ \\
Rash & $2(2.2)$ \\
Nephrotoxicity & $2(2.2)$ \\
Multiple adverse events & $5(5.4)$ \\
Other** & $11(9.3)$ \\
First-line therapy per protocol & $5(4.2)$ \\
Patient-specific factors & $8(6.8)$ \\
Missing data &
\end{tabular}




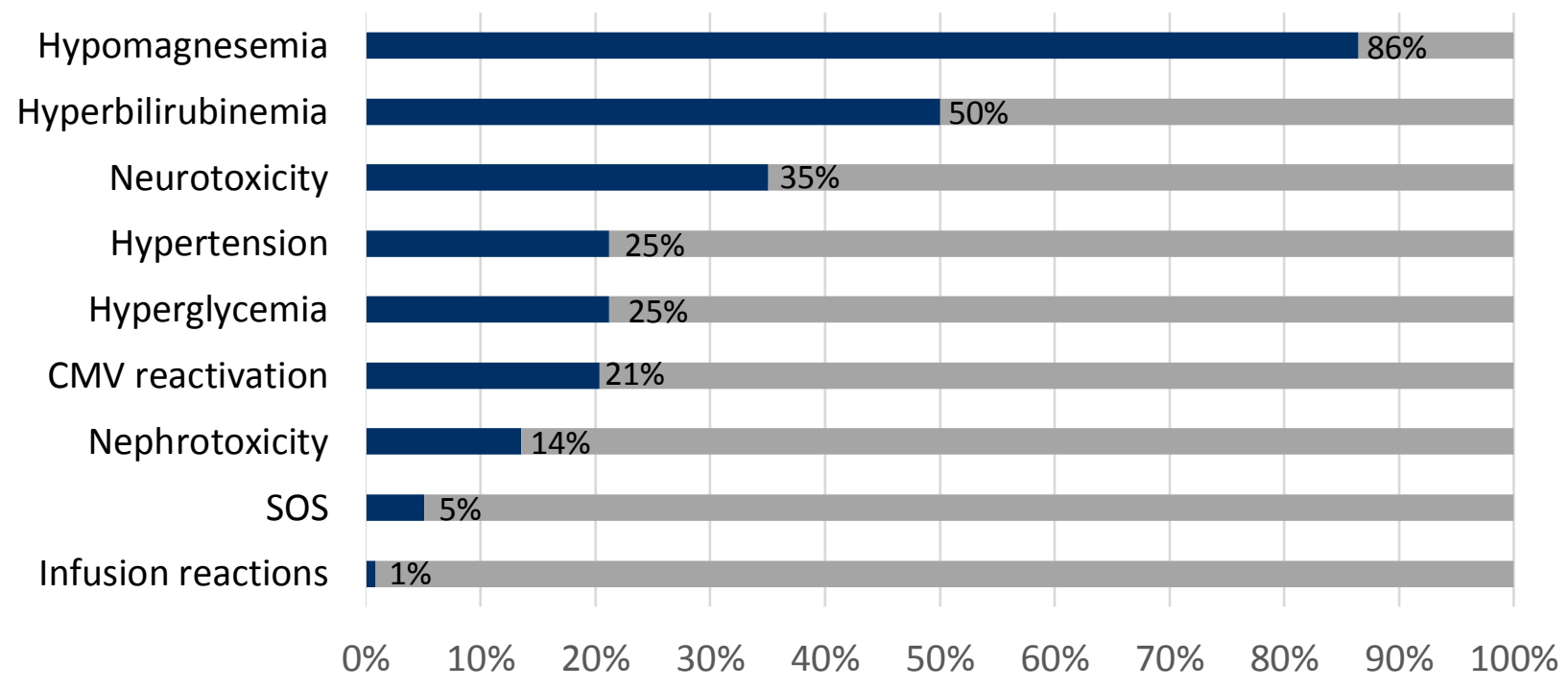

Figure 3. Incidence of Safety Outcomes. $n=118$ for hypomagnesemia, hyperbilirubinemia, hypertension, hyperglycemia, and nephrotoxicity. $n=117$ for neurotoxicity and infusion-related reactions because one paper chart was not available. CMV reactivation and hepatic sinusoidal obstruction syndrome (SOS) are transplantrelated adverse events 


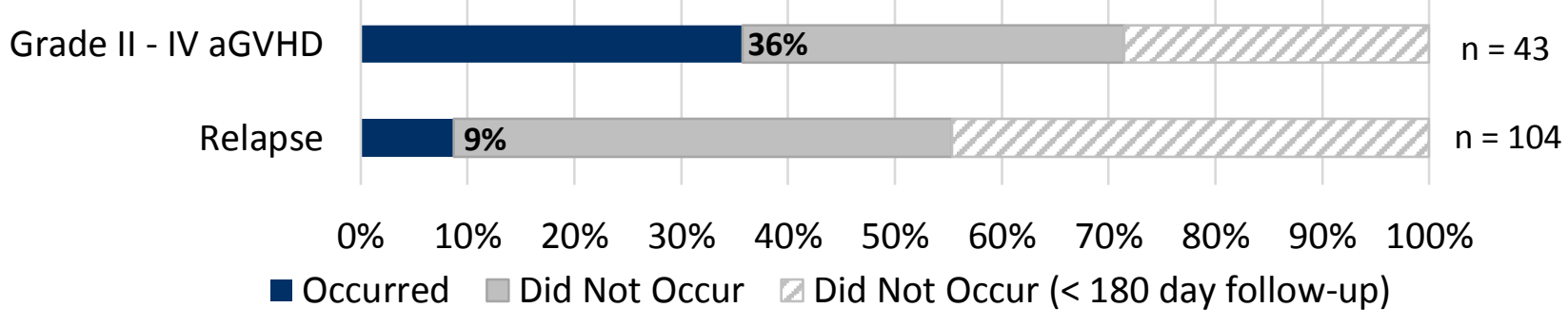

Figure 4. Incidence of Efficacy Outcomes. aGVHD = acute graft-versus-host disease 